\title{
Production and diagnostic application of recombinant domain III of West Nile envelope protein in Brazil
}

\author{
Juliana Helena Chávez ${ }^{[1]}$, Vinicius Pinho dos Reis ${ }^{[1]}$, Jaqueline Raymondi Silva ${ }^{[1]}$, \\ Helen Julie Laure ${ }^{[2],[3]}$, José Cesar Rosa ${ }^{[2],[3]}$, Benedito Antônio Lopes da Fonseca ${ }^{[1]}$ \\ and Luiz Tadeu Moraes Figueiredo ${ }^{[1]}$
}

[1]. Centro de Pesquisa em Virologia, Faculdade de Medicina de Ribeirão Preto, Universidade de São Paulo, Ribeirão Preto, SP. [2]. Centro de Química de Proteínas, Faculdade de Medicina de Ribeirão Preto, Universidade de São Paulo, Ribeirão Preto, SP. [3]. Fundação Hemocentro de Ribeirão Preto, Universidade de São Paulo, Ribeirão Preto, SP.

\begin{abstract}
Introduction: West Nile virus (WNV) is a flavivirus with a natural cycle involving mosquitoes and birds. Over the last 11 years, WNV has spread throughout the Americas with the imminent risk of its introduction in Brazil. Methods: Envelope protein domain III of WNV (rDIII) was bacterially expressed and purified. An enzyme-linked immunosorbent assay with WNV rDIII antigen was standardized against mouse immune fluids (MIAFs) of different flavivirus. Results: WNV rDIII reacted strongly with St. Louis encephalitis virus (SLEV) MIAF but not with other flaviviruses. Conclusions: This antigen may be a potentially useful tool for serologic diagnosis and may contribute in future epidemiological surveillance of WNV infections in Brazil.
\end{abstract}

Keywords: Diagnosis. WNV antibodies. ELISA.

West Nile virus (WNV) is a member of the Flavivirus genus (Flaviviridae), which includes other important arboviruses, such as dengue, yellow fever, Japanese encephalitis, and St. Louis encephalitis viruses ${ }^{1}$. West Nile virus is maintained through a natural cycle involving mosquitoes and birds. The virus also infects humans, horses, and other animals. In humans, WNV can cause illnesses ranging from mild fever to central nervous system infections, including flaccid paralysis and lethal encephalitis. For this reason, WNV is considered as an important public health issue ${ }^{2}$. Historically, outbreaks of WNV have occurred in the Middle East, Europe, and Africa. In 1999, WNV was introduced into North America ${ }^{3}$, and over the last 10 years, it has spread throughout the United States, as well as Canada, Mexico, the Caribbean, and Central and South America $^{4-6}$. Therefore, there is an imminent risk of the WNV introduction in Brazil, and consequently, diagnostic tools are extremely important to perform surveillance and prevent future outbreaks.

West Nile virus is a single-stranded, positive-polarity enveloped ribonucleic acid (RNA) virus with a genome of 11 kilobases, which encodes 10 proteins, three structural and seven nonstructural. The structural proteins include capsid (C), premembrane (prM), and envelope (E) protein. E protein functions in receptor binding, membrane fusion, and viral assembly, and prM assists in proper folding and function

\footnotetext{
Address to: Dr. Luiz Tadeu Moraes Figueiredo. Centro de Pesquisa em Virologia/ FMRP/USP. Av. Bandeirantes 3900, 14049-900 Ribeirão Preto, SP, Brasil. Phone: 5516 3602-3270

e-mail:1tmfigue@fmrp.usp.br; julianachavez@icbim.ufu.br

Received in 20/10/2011

Accepted in 19/01/2012
}

of the E protein ${ }^{7}$. Crystallographic studies reveal that the E protein of flaviviruses folds into three domains: DI, DII, and DIII. In particular, DIII of $\mathrm{E}$ is the putative receptor-binding domain, an important target for neutralizing antibodies and in vivo protection ${ }^{8,9}$. Some authors have suggested that DIII from dengue virus types 1 to 4 (DENV1-4) or japanese encephalitis virus (JEV) could be used as antigens for specific serological diagnosis of flavivirus infections ${ }^{10,11}$. West Nile virus-specific neutralizing epitopes within the structural domain III of the WNV envelope (E) protein also have been identified ${ }^{9}$. Some flaviviruses, such as Saint Louis encephalitis, Japanese encephalitis, dengue and yellow fever, are antigenically related to $\mathrm{WNV}$, and their co-circulation in the same areas has complicated a virus-specific diagnosis of infections in humans and other vertebrate hosts ${ }^{12}$. Therefore, a specific antigen to overcome cross-reaction would be a useful tool in the diagnosis. We show here the production and the preliminary serological tests of a recombinant, bacterially expressed, DIII (r-DIII) peptide from the E protein of WNV.

Inactivated WNV (strain NY-99) was provided by Dr. Mike Drebot (Public Health Agency of Canada, Canadian Science Center). Viral RNA was extracted with QIAamp Extraction kit (QIAGEN, Germany), according to the manufacturer's instructions. DIII was amplified by reverse transcriptase-polymerase chain reaction (RT-PCR) with the following primers (nucleotide positions 1813-2203, GeneBank accession noDQ211652.1): WNV1-forward (GGATCCGTCGGGTCATTTGAAGTGTAGA) and WNV2reverse (AAGCTTGAGGGTGGTTGTAAAGGCTTTG). Restriction sites for Bam HI and Hind III were included in the primer sequence (bold). Briefly, cDNA was generated using the SuperScriptFirst-Strand Synthesis System for RT-PCR 
according to the manufacturer's protocol (Invitrogen, USA). cDNA was used as a template to amplify DIII by PCR using WNV1 and WNV2 primers and Platinum ${ }^{\circledR}$ Taq High Fidelity (Invitrogen, USA). The amplified PCR product was purified from agarose gel with QIAquick Gel Extraction Kit (QIAGEN, Germany) and cloned into the pCR2.1. Topo TA Cloning kit (Invitrogen, USA). Purified pCR2.1 plasmids containing the DIII were sequenced and analyzed. Subsequently, pCR2.1 plasmids and the expression vector PQE31 (QIAGEN, USA) were submitted to a double digestion by Bam HI and Hind III enzymes (Invitrogen, USA) for $4 \mathrm{~h}$. The insert and the expression vector PQE31 were ligated by T4 ligase (Fermentas, USA) during $16 \mathrm{~h}$ at $14^{\circ} \mathrm{C}$ and used to transform M15 Escherichia coli strain cells. Sequence analyses of DIII in the expression vector were performed for further expression of the peptide.

Expression was carried out according to QIA express kit (QIAGEN, USA). Escherichia coli M15 cells were cultured with Luria-Bertani medium containing $100 \mu \mathrm{g} / \mathrm{mL}$ of ampicillin and $25 \mu \mathrm{g} / \mathrm{mL}$ of kanamycin. Protein expression was induced with $1 \mathrm{mM}$ of IPTG at $37^{\circ} \mathrm{C}$ for $4 \mathrm{~h}$. The cells were then harvested and resuspended in lysis buffer $\left(\mathrm{NaH}_{2} \mathrm{PO}_{4} 100 \mathrm{mM}\right.$, Tris-HCL $10 \mathrm{mM}$, and urea $8 \mathrm{M}, \mathrm{pH} 8$ ). A protein with approximately $15 \mathrm{kd}$ after IPTG induction (Figure 1) was observed. Purification was performed with the clarified cell extract mixed with 50\% nickel-nitrilotriacetic acid (Ni-NTA) agarose (QIAGEN, USA) for $2 \mathrm{~h}$ on ice. This mixture was used to load a propylene

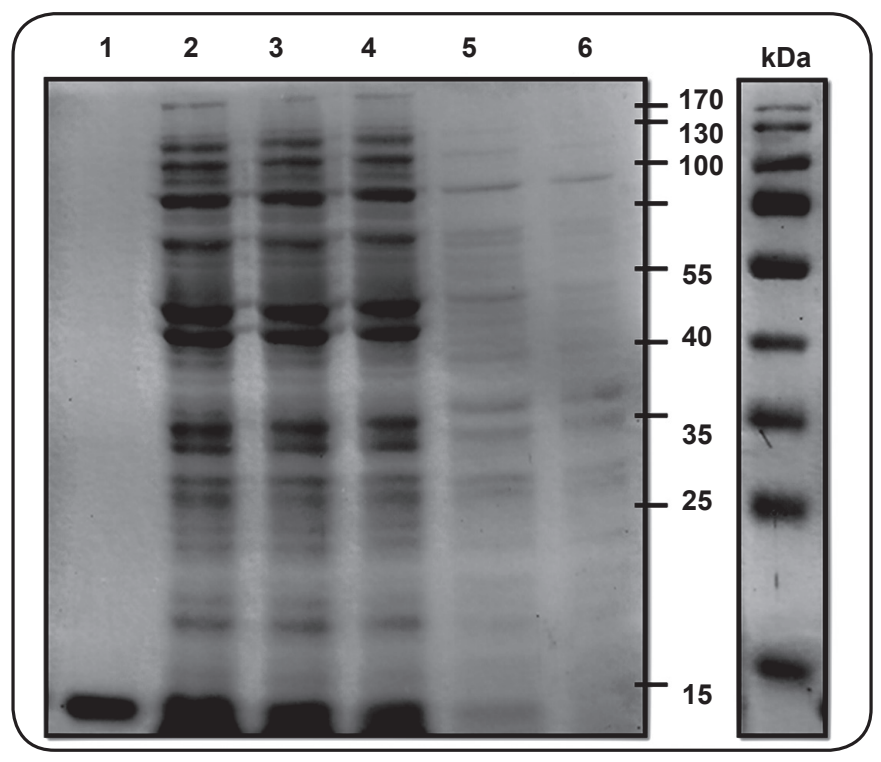

FIGURE 1 - Expression and purification of rDIII-WNV from induced M15 Escherichia coli cells. Protein samples were subjected to $12 \%$ SDS-PAGE and visualized by Coomassie brilliant blue staining.

Lane 1: Elution fraction obtained with elution buffer $\left(\mathrm{NaH}_{2} \mathrm{PO}_{4} 100 \mathrm{mM}\right.$, Tris-HCL $10 \mathrm{mM}$, and urea $8 \mathrm{M}, \mathrm{pH} 4.5$ ) by Ni-NTA purification in propylene columns, presence of a purified protein with $15 \mathrm{kDa}$. Lanes 2-4: Crude extraction from induced M15 Escherichia coli cells with IPTG (1mM) after 4, 3, and 2h, respectively. Lane 5: Flow through fraction. Lane 6: Wash fraction. $\mathrm{kDa}$, kilodaltons based on protein marker PageRuler ${ }^{\mathrm{TM}}$ Prestained Protein Ladder (Fermentas, USA). rDIII: Envelope protein domain III of WNV; WNV: West Nile virus; SDS-PAGE: Sodium dodecyl sulfate-polyacrylamide gel electrophoresis; $\mathbf{N a H}_{2} \mathbf{P O}_{4}$ : sodium dihydrogen phosphate; IPTG: Isopropyl $\beta$-D-1-thiogalactopyranoside column washed extensively with wash buffer $\left(\mathrm{NaH}_{2} \mathrm{PO}_{4}\right.$ $100 \mathrm{mM}$, Tris- $\mathrm{HCl} 10 \mathrm{mM}$, and urea $8 \mathrm{M}, \mathrm{pH} 6.3$ ) and with elution buffer $\left(\mathrm{NaH}_{2} \mathrm{PO}_{4} 100 \mathrm{mM}\right.$, Tris-HCL $10 \mathrm{mM}$, and urea $8 \mathrm{M}, \mathrm{pH} 4.5$ ). Protein purity was assessed using sodium dodecyl sulfate-polyacrylamide gel electrophoresis (SDS-PAGE) in $12 \%$ acrylamide gels (Figure 1). Gels were stained with Coomassie brilliant blue R-250, and the protein content was determined using the reducing agent detergent compatible (RCDC) assay (Bio-Rad, USA) using bovine serum albumin as standard. Protein was characterized using mass spectroscopy (Maldi-TOF-TOF) (data not shown) and by immunoblotting using a human monoclonal immunoglobulin (Ig) M antibody for WNV (Pan-Bio, Australia) (Figure 2).

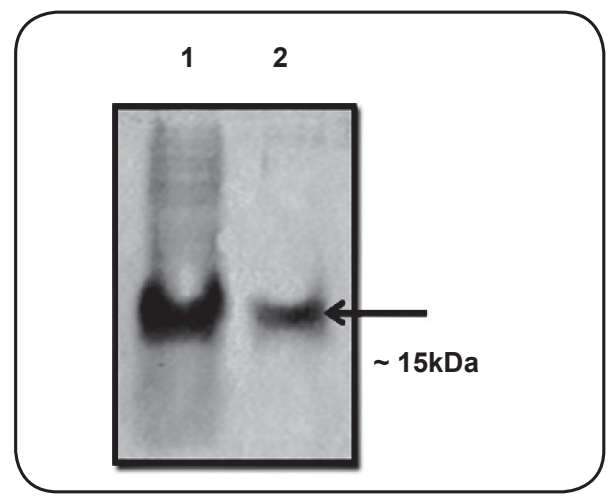

FIGURE 2 - Immunoblotting of rDIII-WNV with IgM WNV monoclonal antibody. Protein samples were subjected to $12 \%$ SDS-PAGE and transferred to nitrocellulose membranes (Bio-Rad, USA) at $100 \mathrm{~V}$ and $4^{\circ} \mathrm{C}$, for $1 \mathrm{~h}$. Blocking was performed for $1 \mathrm{~h}$ (3\% BSA). Membranes were incubated with 1:100 dilution of IgM WNV monoclonal antibody (Pan-Bio, Australia) for $1 \mathrm{~h}$, and bound antibodies were detected using a goat immunoglobulin anti-human IgM conjugated with peroxidase (1:1.000) (Sigma, USA). The reaction was developed with 3,3'-diaminobenzidine (DAB) in a solution also including $0.1 \%$ of $\mathrm{H}_{2} \mathrm{O}_{2}$.

Lane 1: rDIII WNV in crude extract from M15 Escherichia coli-induced cells. Lane 2: rDIII WNV in eluted fraction after Ni-NTA purification in propylene columns. rDIII: Envelope protein domain III of WNV; WNV: West Nile virus; SDS-PAGE: sodium dodecyl sulfate-polyacrylamide gel electrophoresis.

Recombinant enzyme-linked immunosorbent assay (ELISA) with rDIII-WNV antigen was performed as follows. High-binding affinity flat-bottomed 96-well microtiter plates (Corning, USA) were coated with $4 \mu \mathrm{g} / \mathrm{mL}(50 \mu \mathrm{L} /$ well) of rDIII antigen and incubated at $4^{\circ} \mathrm{C}$ during $48 \mathrm{~h}$. Blocking was performed with a $10 \%$ solution of nonfat milk for $2 \mathrm{~h}$ at $37^{\circ} \mathrm{C}$. Mouse immune ascitic fluid (MIAF) of St. Louis encephalitis virus (SLEV) was used as a positive control and as a primary antibody at different dilutions, as well as other flavivirus MIAFs, such as those from Rocio virus (ROCV) Cacipacoré virus (CPCV), which are phylogenetically related to WNV. MIAFs were incubated at $37^{\circ} \mathrm{C}$ for $1 \mathrm{~h}$. An anti-mouse IgG conjugated with peroxidase (Sigma, USA) was used as secondary antibody. The reaction was revealed by adding ABTS (KPL, USA) for $30 \mathrm{~min}$ at $37^{\circ} \mathrm{C}$ and stopped with $\mathrm{H}_{2} \mathrm{SO}_{4} 3 \mathrm{~N}$. Spectrophotometry was performed at $405 \mathrm{~nm}$ in a Multiskan Spectrum (Thermo, USA). Lysates containing the plasmid without the encoding peptide were used as negative antigen control. MIAFs of 
Oropouche virus (OROV) and Mayaro virus (MAYV) were used as primary antibody negative controls. Table 1 shows the absorbance values of the tested MIAFs as preliminary results of this standardized recombinant ELISA.

TABLE 1 - Reactivity of murine polyclonal antibodies (MIAFs) with rDIIIWNV antigen in the standardized IgG-ELISA. rDIII-WNV antigen was used in $4 \mu g / m L$

\begin{tabular}{lcc}
\hline & \multicolumn{2}{c}{ Absorbance in IgG indirect ELISA* } \\
\cline { 2 - 3 } MIAF & WNV rDIII & Purified PQE31 (negative control) \\
\hline SLEV & 1.547 & 0.099 \\
ROCV & 0.088 & 0.082 \\
CPCV & 0.123 & 0.101 \\
OROV & 0.097 & 0.065 \\
MAYV & 0.102 & 0.099
\end{tabular}

*Absorbance values represent the mean of quadruplicate wells. Equivalent values were reached in three independent experiments. SLEV MIAF $(1: 100)$ and other MIAFS were used as primary antibody. Secondary antibody $(1: 2,000)$ was an anti-mouse IgG conjugated with peroxidase (Sigma, USA). Expression vector PQE31 alone was induced and purified to be used as a control for nonspecific binding of antibodies. MIAFs of Oropouche virus and Mayaro virus were used as primary antibody negative controls. MIAFs: mouse immune ascitic fluids; rDIII: envelope protein domain III of WNV; WNV: West Nile virus; IgG-ELISA: immunoglobulin G-enzymelinked immunosorbent assay; PQE31: Expression vector; SLEV: Saint Louis encephalitis virus; ROCV: Rocio virus; CPCV: Cacipacoré virus; OROV: Oropouche virus; MAYV: Mayaro virus.

Considering that linear epitopes in rDIII of flavivirus could bind to antibodies and even promote virus neutralization, the purified denatured rDIII-WNV peptide was used as antigen in an ELISA $^{13}$. Denatured rDIII WNV was used because of the large amount expressed and high purification level. It was observed that the MIAF of SLEV reacted strongly against rDIII-WNV in the test, whereas MIAFs of ROCV and CPCV did not react, although they also are related to WNV. The strong reaction of SLEV MIAF with rDIII-WNV could be explained by the close phylogenetic relationship between these two viruses compared with the other Brazilian flaviviruses ROCV and $\mathrm{CPCV}^{14,15}$. MIAFs of viruses belonging to other viral families, such as OROV (Bunyaviridae) and MAYV (Alphaviridae), also did not react in the recombinant ELISA. A reactivity panel of the tested MIAFs against rDIII-WNV ELISA is shown in Table 1. Unfortunately, it was not possible to test a WNV MIAF in this ELISA because of the lack of this virus in Brazil and, consequently, the restriction in MIAF production. Nonetheless, even considering rDIII-WNV as a suitable antigen in the ELISA, based on the cross-reaction with SLEV MIAF, further investigations might be performed to better establish its utility in this test. A panel of WNV-positive and WNV-negative human and other vertebrate's sera should be used to evaluate the efficacy of this WNV-ELISA. In addition, it would be important to perform a comparison of this standardized ELISA with commercially available tests to assure sensibility and specificity. In short, we have produced an rDIII-WNV antigen that may be useful as antigen in serologic diagnosis of WNV infections. Obviously, further tests must be performed to better characterize this antigen, such as true determination of specificity and sensibility against positive and negative WNV sera. Although those preliminary results strongly indicate that the produced antigen may be used as a national diagnosis resource, in case of the introduction of WNV in Brazil, minimizing the use of expensive imported serologic assays.

\section{CONFLICT OF INTEREST}

The authors declare that there is no conflict of interest.

\section{FINANCIAL SUPPORT}

This research was supported by Fundação de Amparo a Pesquisa de São Paulo (FAPESP), grant 2006/01179-0.

\section{REFERENCES}

1. Murray KO, Walker C, Gould E. The virology, epidemiology, and clinical impact of West Nile virus: a decade of advancements in research since its introduction into the Western Hemisphere. Epidemiol Infect 2011; 139:807-817.

2. Diamond MS, Pierson TC, Fremont DH. The structural immunology of antibody protection against West Nile virus. Immunol Rev 2008; 225:212-225.

3. Petersen LR, Marfin AA, Gubler DJ. West Nile virus. JAMA 2003; 290:524-528.

4. Deardorff E, Estrada-Franco JG, Brault AC, Navarro-Lopes R, CampomanesCortes A, Ramirez-Paz P, et al. Introductions of West Nile virus strains to Mexico. Emerg Infect Dis 2006; 12:314-318

5. Mattar S, Edwards E, Laguado J, Gonzales M, Alvarez J, Komar N. West Nile virus antibodies in Colombian horses. Emerg Infect Dis 2005; 11:1497-1498.

6. Morales MA, Barrandeguy M, Fabbri C, Garcia JB, Vissani A, Trono K, et al. West Nile virus isolation from equines in Argentina, 2006. Emerg Infect Dis 2006; 12:1559-1561.

7. Lindenbach BD, Rice CM. Flaviviridae: The viruses and their replication. In: Knipe DM, Howley PM, editors. Fields Virology. $5^{\text {th }}$ ed. Philadelphia: Lippincott Williams \& Wilkins; 2007. p. 1101-1152.

8. Rey FA, Heinz FX, Mandl C, Kunz C, Harrison SC. The envelope glycoprotein from tick-borne encephalitis virus at 2 Angstrom resolution. Nature 1995; 375:291-298

9. Beasley DW, Barrett AD. Identification of neutralizing epitopes within structural domain III of the West Nile virus envelope protein. J Virol 2002; 76:1309713100 .

10. Fonseca BAL, Khoshnood K, Shope RE, Mason PW. Flavivirus type-specific antigens produced from fusions of a portion of the E protein gene with the Escherichia coli trpE gene. Am J Trop Med Hyg 1991; 44:500-508.

11. SimmonsM,PorterKR,EscamillaJ,GrahamR, WattsDM,EckelsKH,etal.Evaluation of recombinant dengue viral envelope $\mathrm{B}$ domain protein antigens for the detection of dengue complex-specific antibodies. Am J Trop Med Hyg 1998; 58:144-151.

12. Martin DA, Biggerstaff BJ, Allen B, Johnson AJ, Lanciotti RS, Roehrig JT Use of immunoglobulin $m$ cross-reactions in differential diagnosis of human flaviviral encephalitis infections in the United States. Clin Diagn Lab Immunol 2002; 9:544-549.

13. Santos FB, Miagostovich MP, Nogueira RM, Schatzmayr HG, Riley LW, Harris E. Analysis of recombinant dengue virus polypeptides for dengue diagnosis and evaluation of the humoral immune response. Am J Trop Med Hyg 2004; 71:144-152.

14. Figueiredo LTM. The Brazilian flaviviruses. Microbes Infect 2000; 2:1643-1649.

15. Medeiros DB, Nunes MR, Vasconcelos PF, Chang GJ, Kuno G. Complete genome characterization of Rocio virus (Flavivirus: Flaviviridae), a Brazilian flavivirus isolated from a fatal case of encephalitis during an epidemic in Sao Paulo state. J Gen Virol 2007; 88: 2237-2246. 